\title{
MAGNETIC FIELDS ON SOLAR-LIKE STARS: THE FIRST DECADE
}

\author{
S. H. SAAR \\ Harvard-Smithsonian Center for Astrophysics, Mail Stop 58, \\ 60 Garden Street, Cambridge, MA 02138, USA
}

\begin{abstract}
I review the progress made over the past decade in the measurement of magnetic fields on solar-like stars. I describe the evolution of magnetic analysis techniques, summarize our current understanding of stellar magnetic properties, and outline some future research directions.
\end{abstract}

\section{Introduction}

It has now been nearly a decade since Robinson et al. (1980) opened a new era of stellar research by making the first measurements of magnetic fields on solar-like stars. In light of this anniversary it is appropriate to review the field's development and its current analysis techniques, summarize the present state of knowledge in this field, and look ahead to future developments. I also encourage the reader to seek out recent reviews by Saar (1987b), Gray (1988), and Linsky (1989).

The reasons for studying magnetic fields on solar-like stars rise directly from the study of magnetic fields on the Sun. The entire family of atmospheric features on the Sun (plages, spots, granules, prominences, flares, etc.) is related to, or significantly affected by, the presence of magnetic flux. The inferred presence of similar features on cool stars suggests comparable, magnetically-related origins (e.g., Linsky 1985). The million degree corona of the Sun requires magnetic fields for both heating (by direct and indirect means) and confinement of the hot plasma. Observations of stellar coronae, with emission often orders of magnitude larger than the Sun, reinforce the concept that magnetic fields are ubiquitous in cool stars (e.g., Golub 1983). These are but two examples. Clearly, data on magnetic fields is vital to understand fully the physical structure, energy balance and evolution of the stellar atmospheres which the fields permeate.

\section{Evolution of Analysis Methods}

Unfortunately, the solar analogy breaks down when one seeks to employ solar techniques for measuring magnetic fields to the stars. Lack of spatial resolution, combined with the (likely) dipolar structure of the magnetic regions, are the the fundamental problems. They conspire to almost completely cancel stellar circular polarization (e.g., Borra et al. 1984), and reduce

427

J. O. Stenflo (ed.), Solar Photosphere: Structure, Convection, and Magnetic Fields, 427-441.

(c) 1990 by the IAU. 
the linear polarized signal from cool stars to a few parts in $10^{3}$ or less, typically (Huovelin et al. 1988). Magnetic broadening of the unpolarized line profiles remains detectable, but the splitting $\left(\Delta v_{B}\right)$ is, in general, considerably less than the intrinsic line width $(\Delta v)$ at optical wavelengths $\left(\Delta v \approx 4 \mathrm{~km} \mathrm{~s}^{-1}\right.$ at $600 \mathrm{~nm}$ in the Sun, compared with $\Delta v_{B}=$ $1.4 \times 10^{-7} \mathrm{~g}_{\text {eff }} \lambda \mathrm{B}=2 \mathrm{~km} \mathrm{~s}^{-1}$ for $\mathrm{g}_{\text {eff }}=2.5$ and $\mathrm{B}=1 \mathrm{kG}$ ). Furthermore, the Zeeman broadening effect is diluted by the (usually dominant) contributions from non-magnetic regions on the stellar surface. Finally, the lines chosen must be free of blends, and nonmagnetic broadening in the lines should be low. As a result of these requirements, data must have high spectral resolution $\left(\Delta \lambda \leq 2 \Delta \lambda_{B}\right)$, high $\mathrm{S} / \mathrm{N}$ (>100-200), and the star should have $\Delta v_{B} / \Delta v \geq 0.2$ (e.g., $v \sin i \leq 10 \mathrm{~km} \mathrm{~s}^{-1}$ for a $\mathrm{G}$ dwarf at $600 \mathrm{~nm}$ ). Unfortunately, this last constraint does not allow measurement of some of the most interesting, rapidly rotating stars! Some of the above difficulties can be circumvented by observing in the infrared (pioneered by Giampapa et al. 1983) to take advantage of the $\lambda$ dependence of $\Delta v_{B}$. Ultimately, however, the $v \sin i$ constraint is an erent limitation of the method.

Despite these constraints, by the mid-1970's data of the necessary quality could readily be obtained, thanks to advances in electronic detector technology. New analysis methods were now needed. The breakthrough came when Robinson et al. (1980), using the Fourier ratio technique pioneered in solar work by Tarbell and Title (1977), discovered a solar-like field covering a substantial fraction of the active G dwarf, $\xi$ Boo A. Marcy (1982) soon followed with a similar analysis in the wavelength domain. These methods, and all those developed to date, assume that an observed line profile, $\mathrm{F}_{o b s}$, can be interpreted with a two-component model, $\mathrm{F}_{\text {obs }}=\mathrm{fF}_{m}(\mathrm{~B})+(1-\mathrm{f}) \mathrm{F}_{q}(\mathrm{~B}=0)$, where $\mathrm{F}_{m}$ is the profile arising from magnetic regions with a mean field strength $B$ covering a fraction $f$ of the surface, and $\mathrm{F}_{q}$ represents the profile in the field-free $(\mathrm{B}=0)$ regions. Methods differ primarily on how $\mathrm{F}_{m}$ and $\mathrm{F}_{q}$ are computed (or derived) and how the resulting magnetic parameters $\mathrm{f}$ and $\mathrm{B}$ are obtained.

The first analysis methods assumed $\mathrm{F}_{m}$ could be modeled as the appropriately weighted sum (Marcy 1982) or convolution (Robinson 1980) of a triplet of optically thin lines split by a field strength $\mathrm{B}$. The weights reflected the Sears relations for a "disk-averaged" angle $(\gamma)$ between the magnetic field and the line-of-sight (e.g., Marcy 1982, 1984 used $\bar{\gamma}=34^{\circ}$ ). Lines with low $\mathrm{g}_{\text {eff }}$ from the same star were used to simulate these magnetic components and $\mathrm{F}_{q}$. Gray (1984) retained these basic assumptions but extended the Fourier ratio technique to analyze several lines simultaneously by first removing the underlying unsplit profile $\left(=\mathrm{F}_{q}\right)$ of each line through a radiative transfer calculation.

Some problems began to appear, however. The filling factors on many stars seemed unrealistically large (nearly $90 \%$ for the moderately active K dwarf $\epsilon$ Eri, for example). Large magnetic fluxes were recorded for stars with widely different rotation rates and activity levels. Indeed, Gray (1985) showed that the detections made up to that time showed a constant magnetic flux density (i.e., the product, $\mathrm{fB}$ ) for all stars, with the single exception of the Sun. Taken at face value, this result seemed to suggest two possibilities: Either theories regarding the generation of stellar magnetic fields and their roles in heating chromospheres and coronae were wrong, or something was amiss with magnetic measurements.

It now appears that shortcomings in the analysis played an important role in these problems (Hartmann 1987; Saar 1988a; Basri and Marcy 1988). None of the analyses to that time included treatment of radiative transfer effects in the Zeeman components 
themselves; the construction of $\mathrm{F}_{m}$ was made in the optically thin, weak-line limit. This assumption required large filling factors to duplicate observed "saturation" in optically thick line cores, especially in $\mathrm{K}$ dwarfs where the lines employed were generally stronger. When combined with some subtle selection effects (faster rotating, more chromospherically active $\mathrm{G}$ dwarfs and slower rotating, less active $\mathrm{K}$ dwarfs made up most of the sample; see Stepien 1987), these problems led the early analyses to infer $\mathrm{fB}=$ constant.

Clearly, a refined analysis including a radiative transfer model for $\mathrm{F}_{m}$ was needed. Steps in this direction were already taken by Marcy and Bruning (1984), who developed a radiative transfer model to bootstrap comparison between low and high $\mathrm{g}_{\text {eff }}$ lines of substantially different excitation potential. Full integration of magnetic line transfer effects into the analysis was accomplished by Saar and coworkers (Saar and Linsky 1985; Saar et al. 1986a; Saar 1988a), who developed methods which included simple magnetic radiative transfer effects (Unno 1956), the full Zeeman patterns, and some compensation for line blends. These models, however, used convolutions (Gray 1976) to describe velocity broadening, and still required the assumption of an average $\bar{\gamma}$. Bruning (1984) noted that disk integrations are preferred over convolutions for computation of rotational broadening and Saar (1988b) showed use of convolutions could produce errors in $f$ and $B$ values (see also Landolfi et al. 1989). The physics of the line transfer was also quite simple, employing a Milne-Eddington atmosphere with a linear source function and all other variables independent of depth.

These shortcomings have also been addressed recently. Basri and Marcy (1988) and Marcy and Basri (1989) have further improved the analysis by numerically integrating the Unno (1956) equations in realistic model atmospheres (rather than using the MilneEddington approximation). The latest models also employ full disk integration of intensity profiles to obtain the flux (Saar et al. 1989; Marcy and Basri 1989). This step simultaneously eliminates the need to assume an $\bar{\gamma}$, since the angle is automatically accounted for in the disk integration. Known blends can be treated simultaneously by direct line synthesis.

Mathys and Solanki (1989), however, have taken a very different approach. Their technique, based on the solar multi-line regression analysis of Stenflo and Lindegren (1977), correlates line areas measured below the half depth point and the line width at this level with parameters such as excitation potential and a Zeeman broadening term. The Zeeman term, proportional to $\mathrm{fB}^{2}$, can then be separated into $\mathrm{f}$ and $\mathrm{B}$ by comparison with similar studies of the areas and widths for different line depths. Typically, large numbers of lines $(\approx 40)$ are used in the analysis. By sorting lines of varying excitation potential and Zeeman pattern, some crude information on the temperature and average orientation $(\bar{\gamma})$, respectively, of the magnetic regions can potentially be determined. The method is also considerably simpler than detailed model calculations for all the lines. The functional form assumed for the regression equation, however, is physically somewhat unclear, and the choice of its terms is not straightforward. Thus, while the technique appears quite promising, additional calibration and tests are needed. 


\section{A Summary of Current Knowledge}

Using the above techniques, our understanding of stellar magnetic properties has grown steadily over the last decade. In this section I summarize the current knowledge of stellar field strengths and filling factors, and present a fresh analysis of the most recent results.

First, Zeeman broadening is definitely present in measurable quantities on many $\mathrm{G}$ and K dwarfs (e.g., Robinson et al. 1980; Marcy 1984; Gray 1984), often at levels far exceeding that seen in the Sun. It has not yet been observed in F dwarfs (Gray 1984) or in any bright giants or supergiants. It is also usually not seen in subgiants and normal giants (Marcy and Bruning 1984; Gray and Nagar 1985) unless the stars are active RS CVn variables (e.g., Giampapa et al. 1983). And while the presence of Zeeman broadening is small, often debatable, and always difficult to interpret in optical spectra, it is undeniably present in infrared spectra of $M$ dwarfs, where full splitting patterns are visible (Saar and Linsky 1985; Saar et al. 1987). There is also clear evidence for range in Zeeman broadening at each spectral type, since there are clear non-detections for G (Marcy 1984; Gray 1984; Mathys and Solanki 1989), K (Saar 1987a; Saar et al. 1987; Marcy and Basri 1989) and M (Saar et al. 1987) dwarfs as well. Strong magnetic fields have been detected in stars as cool as M4.5 (EV Lac; Saar et al. 1987), indicating that dynamo generation of magnetic flux is still effective even in stars which are almost fully convective.

To explore trends in magnetic parameters $f$ and $B$ in more detail, I have compiled a critically selected group of recent magnetic measurements in Table 1. The list includes virtually all measurements to date which have been derived using radiative transfer methods. This restriction makes the data set more homogeneous, and also excludes probable systematic effects in the earlier measurements (see section 2). In addition, a few optical $M$ dwarf measurements (Bruning et al. 1987) were excluded due to probable blend problems (e.g., Figure 1 of Saar 1988b), and a few results based on lower S/N optical data (in Saar 1987) were also excluded. I included the infrared detections of $\lambda$ And (Giampapa et al. 1983; Gondoin et al. 1986) and the results of Mathys and Solanki (1989). In the latter case, B was computed from the mean of the range given (their table 4), and then I assumed $f \equiv \bar{f}$ $=(\sqrt{\mathrm{f}} \mathrm{B} / \overline{\mathrm{B}})^{2}$. Rotational periods were gathered from Noyes et al. (1984) and Pettersen (1989), and relations in Stepień (1989) and Basri (1987) were used to compute the convective turnover time, $\tau_{c}$. Two measurements are given for a single star in several cases where different investigators disagreed on the magnetic parameters (e.g., HD $131156 \mathrm{~A}=\xi$ Boo A). When this occurs, both are plotted and connected with a dotted line.

One of the first things apparent is a general tendency for magnetic field strengths to increase with increasing B-V (i.e., towards later spectral types). This trend, combined with the lack of detections for $\mathrm{F}$ dwarfs and most giants and subgiants (likely due to low B; Marcy and Bruning 1984), and the low field strengths seen on the active giant, $\lambda$ And, strongly suggests a relationship between $\mathrm{B}$ and some intrinsic property of the stellar atmosphere. Saar and Linsky (1986a) found a tight correlation between $\mathrm{B}$ and the equipartition "pressure-balancing" magnetic field, $\mathrm{B}_{e q} \propto \mathrm{P}_{\text {gas }}^{0.5}$, where $\mathrm{P}_{\text {gas }}$ is the photospheric gas pressure. I estimated $\mathrm{P}_{\text {gas }}$ as a function of $\mathrm{T}_{\text {eff }}$ and gravity by using atmospheres of Kurucz (1979 and unpublished), supplemented with models derived by Mould (1976) for M dwarfs. The gas pressure was determined at optical depth, $\tau_{5000}=1$, where $\mathrm{P}_{\text {gas }}(\odot)$ yields $\mathrm{B}_{e q} \approx 1.5$ $\mathrm{kG}$ (note that the exact choice of $\tau_{5000}$ is not critical, since we use $\mathrm{P}_{\text {gas }}$ only to scale relative 
Table 1: A Critical Selection of Recent Magnetic Field Determinations

\begin{tabular}{|c|c|c|c|c|c|c|c|c|}
\hline $\begin{array}{l}\text { Star } \\
\text { ID }\end{array}$ & Sp. Type & $\begin{array}{c}\mathrm{B}-\mathrm{V} \\
(R-I)\end{array}$ & $\begin{array}{c}\mathrm{B} \\
(\mathrm{kG})\end{array}$ & $\begin{array}{c}\mathrm{f} \\
(\%)\end{array}$ & Ref. & $\begin{array}{c}\text { adopted } \\
\mathrm{B}_{e q}\end{array}$ & $\begin{array}{c}\mathrm{P}_{\text {rot }} \\
\text { (days) }\end{array}$ & $\begin{array}{c}\tau_{c} \\
\text { (days) }\end{array}$ \\
\hline HD 39587 & G0V & 0.59 & 1.0 & 60 & 1 & 1.4 & 5.2 & 10.0 \\
\hline HD 190406 & G1V & 0.61 & 1.8 & 10 & 1 & 1.4 & 13.5 & 11.0 \\
\hline HD 1835 & G2V & 0.66 & 1.4 & 32 & 1 & 1.5 & 7.7 & 13.7 \\
\hline HD 28099 & G6V & 0.66 & 1.7 & 30 & 2 & 1.5 & 8.7 & 13.7 \\
\hline HD 20630 & G5V & 0.68 & 1.5 & 35 & 1 & 1.6 & 9.4 & 14.7 \\
\hline HD 10700 & G8V & 0.72 & $(\sqrt{\mathrm{f}} \mathrm{B}$ & $\leq 0.2)$ & 12 & 1.7 & 31.9 & 16.9 \\
\hline \multirow[t]{2}{*}{ HD $131156 \mathrm{~A}$} & G8V & 0.76 & 1.6 & 22 & 3 & 1.7 & 6.2 & 19.0 \\
\hline & G8V & 0.76 & 1.8 & 35 & 1 & 1.7 & 6.2 & 19.0 \\
\hline HD 152391 & G8V & 0.76 & 1.7 & 18 & 1 & 1.7 & 11.1 & 19.0 \\
\hline HD 3651 & $\mathrm{~K} 0 \mathrm{~V}$ & 0.85 & $\ldots$ & $\ldots$ & 3 & 1.9 & 48 & 20.5 \\
\hline HD 10476 & $\mathrm{~K} 1 \mathrm{~V}$ & 0.84 & 1.0 & 17 & 3 & 2.0 & 38 & 20.5 \\
\hline HD 165341 & $\mathrm{~K} 1 \mathrm{~V}$ & 0.86 & 1.2 & 18 & 3 & 2.0 & 19.7 & 20.5 \\
\hline HD 155885 & $\mathrm{~K} 1 \mathrm{~V}$ & 0.86 & 1.5 & 13 & 3 & 2.0 & 22.9 & 20.5 \\
\hline \multirow[t]{2}{*}{ HD 22049} & $\mathrm{~K} 2 \mathrm{~V}$ & 0.88 & 1.0 & 30 & 3 & 2.2 & 11.3 & 20.5 \\
\hline & $\mathrm{K} 2 \mathrm{~V}$ & 0.88 & 1.9 & 12 & 4 & 2.2 & 11.3 & 20.5 \\
\hline HD 115404 & $\mathrm{~K} 2 \mathrm{~V}$ & 0.93 & 2.1 & 20 & 1 & 2.2 & 18.8 & 20.5 \\
\hline HD $45088 \mathrm{~A}$ & $\mathrm{~K} 3 \mathrm{Ve}$ & 0.96 & 2.4 & 50 & 2 & 2.4 & 7.4 & 20.5 \\
\hline HD 209100 & $\mathrm{~K} 4-5 \mathrm{~V}$ & 1.09 & 2.6 & 13 & 12 & 2.5 & $\ldots$ & 20.5 \\
\hline HD 225732 & $\mathrm{~K} 6 \mathrm{~V}$ & 1.04 & 1.8 & 20 & 7 & 2.5 & $\ldots$ & 20.5 \\
\hline HD $131156 \mathrm{~B}$ & $\mathrm{~K} 4 \mathrm{~V}$ & 1.10 & (if 2.6 & $\leq 20)$ & 1 & 2.6 & 11.5 & 20.5 \\
\hline EQ Vir & $\mathrm{K} 5 \mathrm{Ve}$ & 1.18 & 2.5 & 80 & 5 & 2.7 & 3.9 & 20.5 \\
\hline \multirow[t]{2}{*}{ HD 201091} & $\mathrm{~K} 5 \mathrm{~V}$ & 1.18 & (if 1.5 & $\leq 5)$ & 1,6 & 2.7 & 37.9 & 20.5 \\
\hline & $\mathrm{K} 5 \mathrm{~V}$ & 1.18 & 1.2 & 24 & 3 & 2.7 & 37.9 & 20.5 \\
\hline BY Dra & $\mathrm{K} 5 \mathrm{Ve}+$ & 1.19 & 2.8 & 60 & 8 & 2.7 & 3.8 & 20.5 \\
\hline HD 201092 & $\mathrm{~K} 7 \mathrm{~V}$ & 1.38 & (if 1.5 & $\leq 10)$ & 8 & 2.9 & 48.0 & 20.5 \\
\hline HD 97101 & K9V & 1.35 & 1.8 & 25 & 7 & 2.9 & $\ldots$ & 20.5 \\
\hline \multirow[t]{2}{*}{ HD 88230} & $\mathrm{~K} 7 \mathrm{~V}$ & 1.37 & (if 1.5 & $\leq 10)$ & 8 & 2.9 & $\ldots$ & 20.5 \\
\hline & $\mathrm{K} 7 \mathrm{~V}$ & 1.37 & 0.8 & 55 & 7 & 2.9 & $\ldots$ & 20.5 \\
\hline GL 205 & $\mathrm{M} 1.5 \mathrm{~V}$ & $(0.85)$ & (if 1.5 & $\leq 15)$ & 8 & 3.4 & $\ldots$ & 20.5 \\
\hline AU Mic & $\mathrm{M} 1.5 \mathrm{Ve}$ & $(0.84)$ & 4.0 & 90 & 8 & 3.4 & 4.8 & 20.5 \\
\hline$=\mathrm{AD} \mathrm{Leo}$ & M3.5Ve & $(1.12)$ & 3.8 & 73 & 6,8 & 3.8 & 2.7 & 20.5 \\
\hline GL 273 & M4V & (1.15) & (if 1.5 & $\leq 25)$ & 8 & 4.0 & $\ldots$ & 20.5 \\
\hline EV Lac & $\mathrm{M} 4.5 \mathrm{Ve}$ & $(1.15)$ & 5.2 & 90 & 8 & 4.4 & 4.4 & 20.5 \\
\hline \multirow[t]{2}{*}{ HD 222107} & G8III-IV & 1.01 & 0.6 & 20 & 9 & 0.6 & 20.5 & 87.3 \\
\hline & G8III-IV & 1.01 & 1.2 & 48 & 10 & 0.6 & 20.5 & 87.3 \\
\hline HD 17433 & K1-2IVe & 0.96 & 2.0 & 60 & 11 & 1.3 & 12.1 & 89.6 \\
\hline
\end{tabular}

References: ${ }^{1}$ Saar (1987); ${ }^{2}$ Saar and Linsky (1986); ${ }^{3}$ Basri and Marcy (1989); ${ }^{4}$ Saar et al. (1986b); ${ }^{5}$ Saar et al. (1986a); ${ }^{6}$ Saar and Linsky (1985); ${ }^{7}$ Bruning et al. (1987); ${ }^{8} \mathrm{Saar}$ et al. (1987); ${ }^{9} \mathrm{Gondoin}$ et al. (1986); ${ }^{10} \mathrm{Giampapa}$ et al. (1983); ${ }^{11} \mathrm{Bopp}$ et al. (1989); ${ }^{12}$ Mathys and Solanki (1989) 
to the Sun). The resulting $\mathrm{B}_{e q}$ was then determined using $\mathrm{B}_{e q}=\left(\mathrm{P}_{\text {gas }} / \mathrm{P}_{\text {gas }}(\odot)\right)^{0.5} \mathrm{~B}(\odot)$. The results (with $\mathrm{B}_{\odot} \equiv 1.5 \mathrm{kG}$; Harvey and Hall 1975) are given in Table 1 ; for $\mathrm{G}$ and $\mathrm{K}$ stars they are very similar to $\mathrm{B}_{e q}$ derived by Zwaan and Cram (1989).

$\mathrm{A}$ plot of $\mathrm{B}$ and $\mathrm{B}_{e q}$ (Figure 1) shows most stars clustering near or below the $\mathrm{B}=\mathrm{B}_{e q}$ line. I interpret this as evidence that magnetic field strengths in stellar photospheres are largely determined by a horizontal pressure balance in which gas pressure dominates, i.e., B $\leq \mathrm{B}_{e q} \propto \mathrm{P}_{g a s}^{0.5}$. Several theories of magnetic field concentration in stellar photospheres predict this type of relation (e.g., Parker 1978; Spruit and Zweibel 1979; Galloway and Weiss 1981). One RS CVn data point lies considerably above the $\mathrm{B}=\mathrm{B}_{e q}$ line (Giampapa et al. 1983); Giampapa (1984), however, postulates that the stellar surface had an admixture of umbrae (with much higher B) in the field of view during the observation. In the case of the second RS CVn (HD 17433), which also shows a rather large field strength, enhanced turbulence may play a larger role in the pressure balance (Bopp et al. 1989).

Hartmann's (1987) suggestion that the $\mathrm{B} \propto \mathrm{B}_{e q}$ relation might be due to line opacity effects is probably not correct, since the analyses used to derive the data in Table 1 explicitly take opacity broadening into account. His comment that stars with low B may be missed due to difficulties in detection (e.g., Marcy 1982; Saar 1988a), however, is certainly valid. An equally important problem is the following: How valid it is to mix results of data

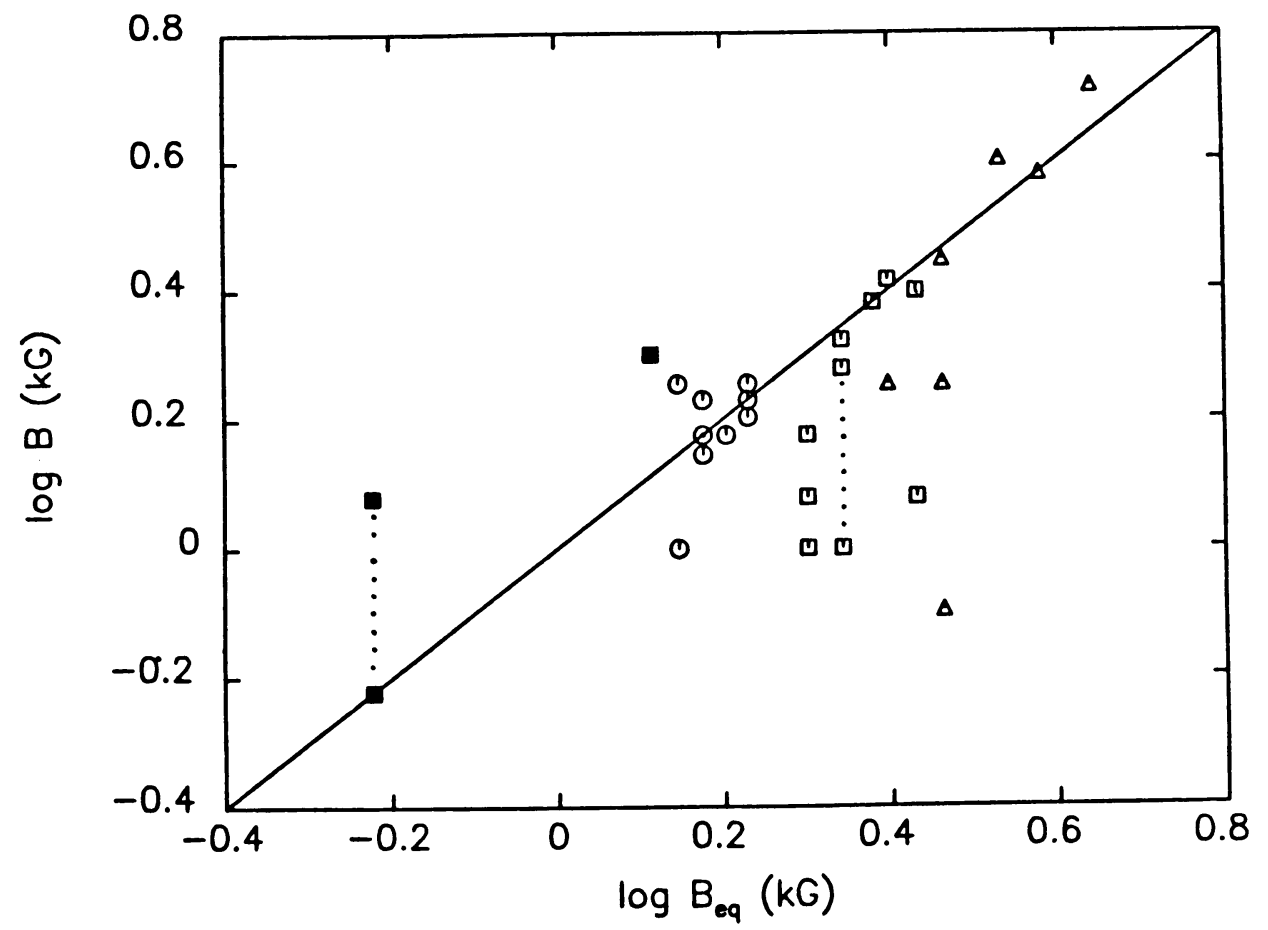

Figure 1. $\mathrm{B}$ versus $\mathrm{B}_{e q} \propto \mathrm{P}_{\text {gas }}^{0.5}$. Circles, squares, triangles, and filled squares represent $\mathrm{G}$, $\mathrm{K}, \mathrm{M}$ dwarfs and RS CVn variables, respectively. The Sun is indicated by $\odot$. The relation $\mathrm{B}=\mathrm{B}_{e q}$ is shown as a solid line. $\mathrm{B} \leq \mathrm{B}_{e q}$ is inferred from the data. 
sets measured using different lines and wavlengths? Table 1 contains magnetic parameters derived from medium strength Ti I lines at $2200 \mathrm{~nm}$ (e.g., Saar and Linsky 1985), Fe I lines at $617 \mathrm{~nm}$ (e.g., Saar et al. 1986a) and $1600 \mathrm{~nm}$ (Gondoin et al. 1986) and strong Fe I lines at $846 \mathrm{~nm}$ (Marcy and Basri 1989). Continuum opacity (primarily $\mathrm{H}^{-}$) is similar at 617 and $2200 \mathrm{~nm}$, but is stronger at $846 \mathrm{~nm}$ and substantially weaker at $1600 \mathrm{~nm}$ (I thank S. Solanki for pointing this out). If all other variables are held constant, fields should appear stronger (with a lower f) at $1600 \mathrm{~nm}$, where the observer sees deep into the atmosphere, and weaker (with higher $\mathrm{f}$ ) at $846 \mathrm{~nm}$, where the observer sees deep into the atmosphere. Indeed, the $\mathrm{B}$ values derived for $\mathrm{K}$ dwarfs using the $846 \mathrm{~nm}$ line (Marcy and Basri (1989) are somewhat smaller (and the $f$ values larger) than those of derived using $617 \mathrm{~nm}$. The considerably greater strength of the $846 \mathrm{~nm}$ feature will make some parts of its profile more sensitive to higher atmospheric levels (with lower $B$ and higher $f$ values) than $617 \mathrm{~nm}$. Similarly, optical determinations of B differ from those at $1600 \mathrm{~nm}$ by $300-400 \mathrm{G}$ in the Sun (e.g., Stenflo 1989). Thus, it is possible that some of the variation in measured field strengths in $\mathrm{K}$ dwarfs is due to the effects of differing line formation heights. Of course, differences in the analysis methods used will also affect the results. A more detailed analysis, using flux tube models (e.g., Steiner et al. 1986) to determine the contribution functions (e.g., Grossmann-Doerth et al. 1988) for magnetic lines at different wavelengths, will probably be needed to solve this puzzle.

A search for other correlations with $B$ yields little. Plots of $B$ versus $f$ show no correlation (see also Saar and Linsky $1986 \mathrm{a}$ ), verifying that $\mathrm{fB}$ is not a constant. Also, $\sqrt{\mathrm{fB}}$ is not constant, as would be expected if errors in the separation of $f$ and $B$ were dominant (Gray 1984; Saar 1988a). No clear relation exists between B and rotation, either. Plots of B with the inverse Rossby number, $\tau_{c} \Omega$, are basically flat. There appears to be a weak relationship between $\mathrm{B}$ and $\Omega\left(\mathrm{B} \propto \Omega^{0.3 \pm 0.2}\right.$; see also Saar $\left.1987 \mathrm{a}\right)$, but this probably represents a selection effect (only stars with large $B$ can be detected at large $\Omega$; Saar 1988a).

The lack of a B-rotation correlation is at first suprising; many simple dynamo models predict $\mathrm{B} \propto \Omega$ or $\tau_{c} \Omega$ (e.g., Stix 1972). However, when it is understood that dynamo theories actually predict the magnetic flux generated, rather than field strength, the paradox is resolved. Marcy (1984) and Gray (1985)noted the first indications that magnetic flux measurements and stellar rotation were related. Using mostly older data, Stepień (1987) found magnetic flux correlated with $\left(\tau_{c} \Omega\right)^{0.5}$. In the present data set, magnetic flux density (fB) exhibits strong correlations with the stellar rotation: $\mathrm{fB} \propto \Omega^{1.3}$ and $\mathrm{fB} \propto\left(\tau_{c} \Omega\right)^{1.2}$ (Figure 2; see also Saar 1987a,b). These relations are consistent with the $\mathrm{fB} \propto \mathrm{T}_{\text {eff }}{ }^{-n} \Omega$ relation correlation found by Marcy and Basri (1989), and are broadly consistent with many simple theoretical expectations (e.g., Schatzman 1962). The Durney and Robinson (1982) dynamo model, however, which predicts $f \propto \Omega^{2.5}$, appears to be ruled out.

Since $B$ is not strongly correlated with rotation, the filling factor must be the cause for the $\mathrm{fB}$ - rotation relations. A plot of $\mathrm{f}$ versus $\tau_{c} \Omega$ confirms this (Figure 3). A least-squares fit to the Table 1 data set yields $f \propto\left(\tau_{c} \Omega\right)^{0.9}$ (see Linsky and Saar 1987; Saar 1987b; Saar et al. 1987; Stepien 1988a). The filling factor must be less than one by definition, so it is inevitable that this simple power law breaks down at some point and $f$ "saturates". Based on the derived power law, stars beyond $\tau_{c} \Omega \approx 0.8$ may be in this state (see also Saar and Linsky 1986a; Linsky and Saar 1987). Thus, a dynamo model including a saturated state (e.g., Skumanich and MacGregor 1986) fits the data best. Saturated states in chromospheric 

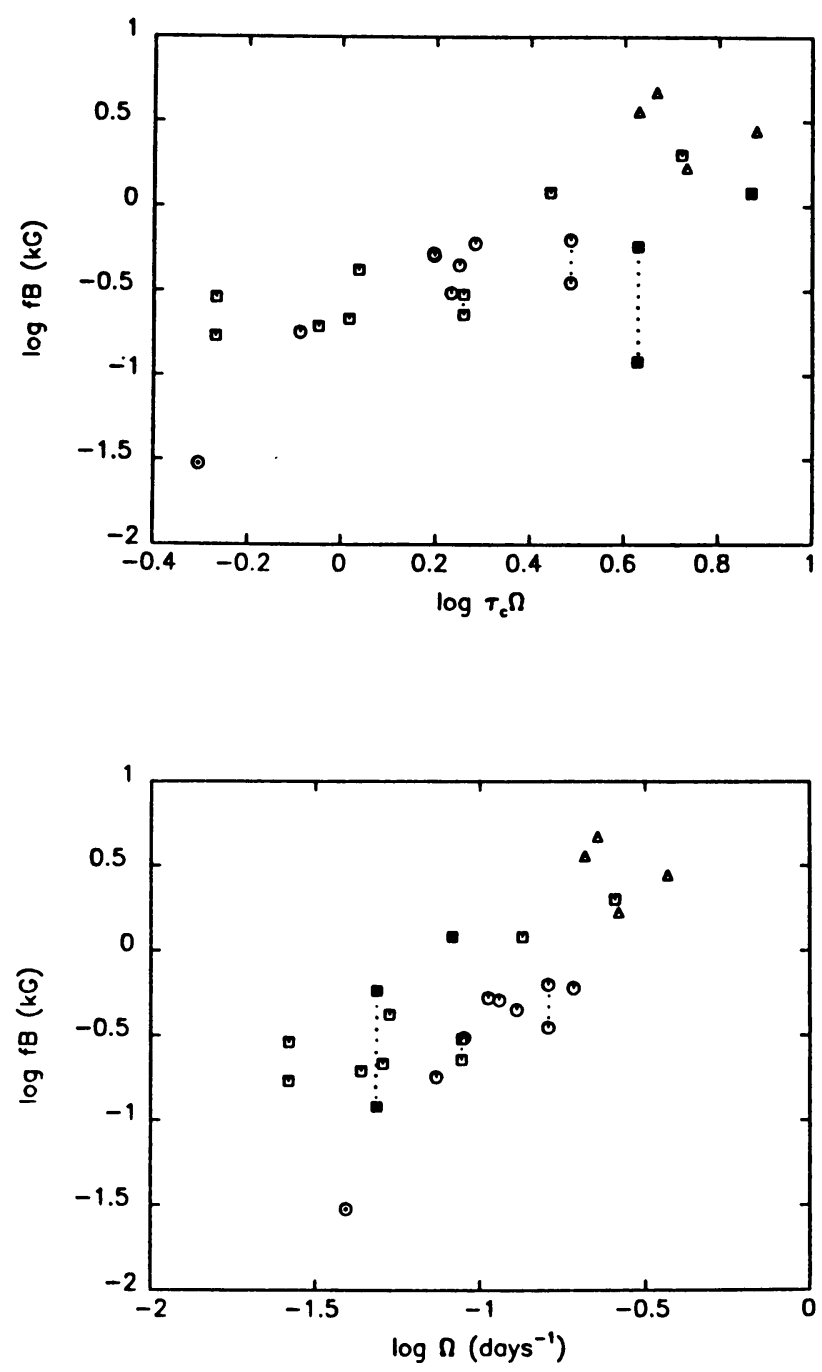

Figure 2. Magnetic flux density (fB) versus rotational parameters. Symbols are same as in Fig. 1. Magnetic flux density correlates with $\tau_{c} \Omega \quad\left(\mathrm{fB} \propto\left(\tau_{c} \Omega\right)^{1.2 \pm 0.1}\right.$; top) and $\Omega$ $\left(\mathrm{fB} \propto \Omega^{1.3 \pm 0.1}\right.$; bottom) largely the result of correlations between $\mathrm{f}$ and rotation (see Fig. 3 ). 


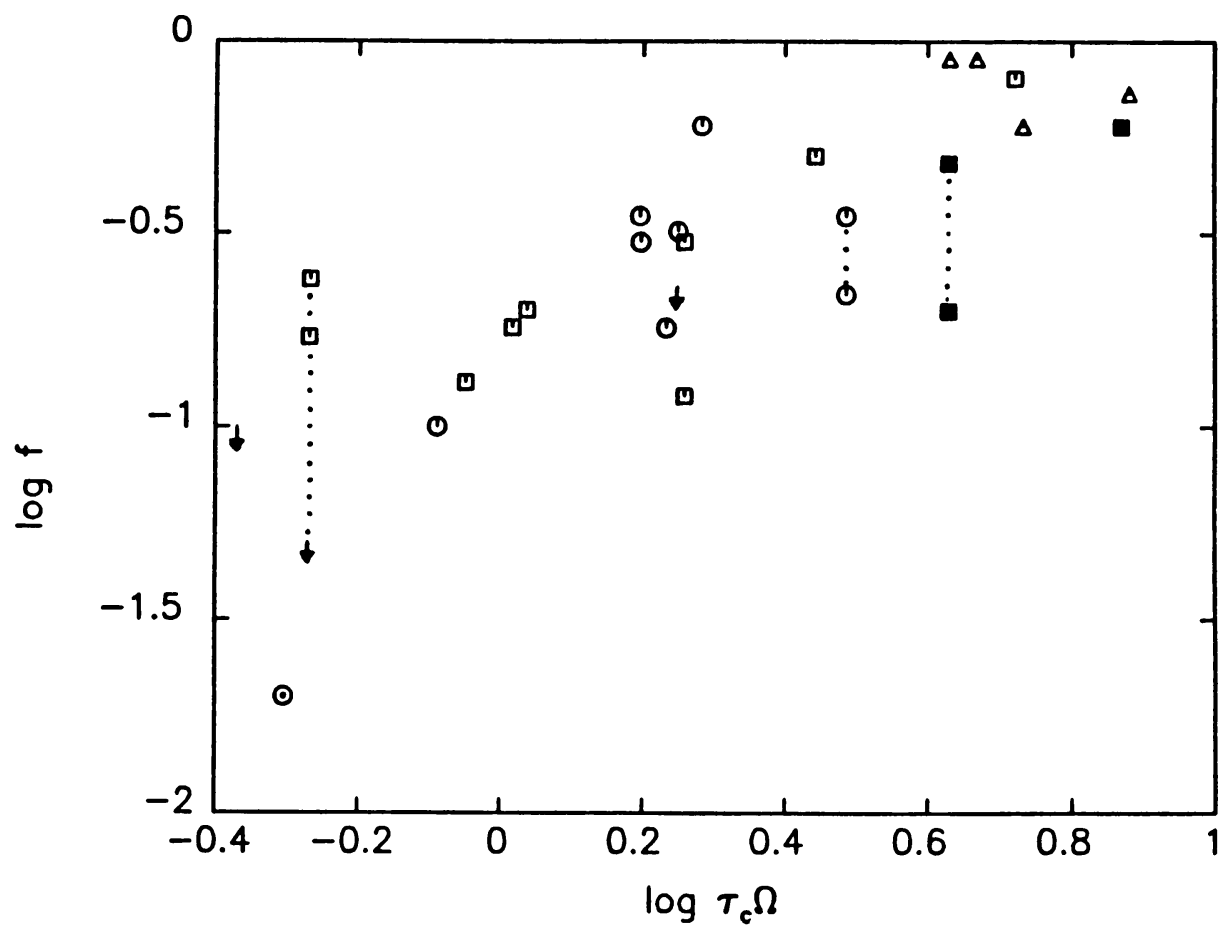

Figure 3. Magnetic filling factor $\mathrm{f}$ versus $\tau_{c} \Omega$. Symbols are as in Fig. 1, with upper limits to $f$ indicated by arrows. A least-squares fit yields $f \propto\left(\tau_{c} \Omega\right)^{0.9 \pm 0.2}$. The magnetic filling factor appears to be the dominant magnetic parameter controlling rotation-activity and rotation-age relations.

and coronal emission have been recognized for some time (e.g., Vilhu 1984).

As one might anticipate based on the $\mathrm{fB}$ - rotation correlations, there are also indications (though the data set is tiny) that $\mathrm{fB}$ declines with stellar age (Linsky and Saar 1987; Saar 1989). The magnetic field strength versus age diagram shows only scatter, implying that a reduction in the surface filling factor of active regions is the primary reason for the decline in stellar activity with time (at least for ages $\geq 0.3 \mathrm{Gyr}$ ). The decline of $\mathrm{fB}$ with time increases with time, but the functional form cannot be determined precisely with the available data. This result, too, is broadly consistent with theoretical models of angular momentum loss (e.g., Weber and Davis 1967; Kawaler 1988; Stepien 1988b).

Unlike the field strength, $f$ exhibits a large range independent of color. Flare stars (listed by variable star name in Table 1) appear to have the highest filling factors. The $\mathrm{f}$ values of $\mathrm{dM}$ stars suggests that the large $\mathrm{H} \alpha$ filling factors derived by Giampapa (1985) refer to the chromospheric level, where $f$ is indeed much larger than in the photosphere (Saar et al. 1987). Filling factors estimated by Montesinos et al. (1987) are generally much smaller than those observed.

Due to the intimate connection between chromospheric, transition-region, and coronal heating and magnetic fields on the Sun, it is natural to search for similar relations in the 
stellar data. Marcy (1984), once again, lead the way, noting correlations between magnetic parameters, Ca II, and X-ray emission. Saar and Schrijver (1987) found $\mathrm{F}_{x} \propto(\mathrm{fB})^{0.9}$ and $\Delta \mathrm{F}_{C a I I} \propto(\mathrm{fB})^{0.6}$ for $\mathrm{fB} \leq 300 \mathrm{G}$ (where $\Delta \mathrm{F}_{C a I I}$ is the residual Ca II flux; Schrijver 1983). Above $\mathrm{fB}=300 \mathrm{G}$, they found $\Delta \mathrm{F}_{C a I I}$ was saturated. Schrijver et al. (1989) note that these correlations are consistent with relations derived for the Sun, and with flux-flux relations derived for stars. Saar (1988c) derived power law relations (with ranging from 0.6 and 0.3) between fB and ultraviolet C IV, C II and O I line fluxes. Quillen et al. (1987) found that using X-ray luminosities and the simple coronal loop model of Golub (1983), they could roughly predict stellar magnetic fluxes to within a scaling factor. Estimates of the individual $f$ and $B$ values were rather poor, however. Stepien (1988a) and Jordan et al. (1987) have predicted expected magnetic flux - activity relations with some success (e.g., Jordan et al. estimate $\mathrm{F}_{X} \propto(\mathrm{fB})^{1.8}$ ).

Groups have also searched for variability in stellar magnetic fields. Already in the first detections, Robinson et al. (1980) noted an apparent change on $\xi$ Boo A. Subsequent observations of the star by Marcy (1981) and Gondoin et al. (1986) yielded no evidence for magnetic line broadening at all, suggesting the star had a large range of magnetic variation (consistent with its $\mathrm{Ca}$ II emission). Rotational modulation of chromospheric and transition-region line fluxes with magnetic flux for the active dwarf $\xi$ Boo A support this picture (Saar et al. 1988). The same authors used simultaneous measurements of broadband linear polarization (which measures the net tangential component of the magnetic field) to permit a rough determination of the spatial distribution of active areas on the star. Some of this variability may have been due to changes in turbulence due to a "starpatch", as postulated by Toner and Gray (1988). On the other hand, it is possible (Bruning and Saar 1989) that the line bisector variations seen in both data sets are primarily a magnetic phenomenon (masked in the Toner and Gray analysis by the small $\mathrm{g}_{e f f}$ leverage: $\mathrm{g}_{e f f}=$ 1.0 versus $\left.g_{e f f}=1.7\right)$. More work on the interaction of magnetic fields and convection, and their role in line shapes, is certainly needed.

Other stars studied in detail so far seem not to vary as much as $\xi$ Boo A. Basri and Marcy (1988) found little change in their $\epsilon$ Eri spectra, confirming earlier evidence (Saar et al. 1986b) that its variability range is small. The active subgiant HD 17433 (see Bopp et al. 1989) has also been the subject of a campaign of simultaneous magnetic field, ultraviolet, and optical measurements (Ambruster and Saar 1988). Little variability was observed here either, except during a brief flare, when C IV emission increased significantly and there was weak evidence for a change in the magnetic parameters. Analysis of a similar campaign on the nearly pole-on flare star BD +26 730 is underway (Saar et al. 1989, in preparation).

\section{A Comparison of $\epsilon$ Eri Measurements}

As an example of progress in magnetic measurements and difficulties yet to be resolved, Table 2 shows a compilation of all magnetic measurements to date for the active $\mathrm{K} 2$ dwarf, $\epsilon$ Eri (HD 22049). All filling factors were adjusted (where possible) to the scale of Marcy $\left(1984 ; \bar{\gamma}=34^{\circ}\right)$.

Clearly, there has been some evolution of opinion as to the magnetic activity on the star, and the situation is perhaps still not clearly resolved. Early approaches which did not include radiative transfer effects in the Zeeman process (Marcy 1984; Gray 1984) generally 
Table 2: Comparison of Magnetic Measurements for the K2 dwarf, $\epsilon$ Eridani

\begin{tabular}{|l|cccccc|}
\hline Reference & $\begin{array}{c}\mathrm{B} \\
(\mathrm{kG})\end{array}$ & $\begin{array}{c}\mathrm{f} \\
(\%)\end{array}$ & $\begin{array}{c}\mathrm{fB} \\
(\mathrm{kG})\end{array}$ & $\begin{array}{c}\sqrt{\mathrm{fB}} \\
(\mathrm{kG})\end{array}$ & $\begin{array}{c}\lambda \\
(\mathrm{nm})\end{array}$ & $\begin{array}{c}\text { \# of lines } \\
\mathrm{g}<1.3 / \mathrm{g} \geq 1.3\end{array}$ \\
\hline Marcy (1984) & 1.17 & 67 & 0.78 & 0.96 & 617 & $1 / 1$ \\
Gray (1984) & 1.9 & 36 & 0.69 & 1.14 & 640 & $10 / 6$ \\
Saar et al. (1986b) & 1.9 & 13 & 0.25 & 0.69 & 617 & $3 / 2$ \\
Saar (1988) & $($ if 2.0 & $\leq 5)$ & $\leq 0.10$ & $\leq 0.40$ & 2210 & $1 / 1$ \\
Saar (1988) & $3.0:$ & $8:$ & $0.24:$ & $0.85:$ & 617 & $3 / 2$ \\
Mathys and Solanki (1988) & $1.79-2.53$ & $10-20$ & $(0.30)$ & 0.80 & $580-680$ & $(45$ total) \\
Basri and Marcy (1988) & 1.0 & 35 & 0.35 & 0.59 & 846 & $1 / 1$ \\
Mathys and Solanki (1989) & $2.09-2.96$ & $10-20$ & $(0.35)$ & 0.94 & $580-680$ & $33 / 9$ \\
Marcy and Basri (1989) & 1.0 & 30 & 0.30 & 0.55 & 846 & $1 / 1$ \\
Marcy and Basri (1989) & $($ if 3.0 & $\leq 10)$ & $\leq 0.30$ & $\leq 0.95$ & 2210 & $1 / 0$ \\
& (if 1.0 & $\leq 30)$ & $\leq 0.30$ & $\leq 0.55$ & 2210 & $1 / 0$ \\
\hline
\end{tabular}

produced higher filling factors. As noted in section 2, this is likely due to the need for higher $f$ to mimic the more "box-shaped" (due to increased line opacity) doppler core of the line, in the absence of a radiative transfer treatment (e.g., Saar 1988a). Introduction of a simple (Unno 1956) radiative transfer model led to smaller filling factors (Saar et al. 1986b; average of 11 measurements). Later, analysis of simultaneous optical and infrared spectra using the same model indicated lower fluxes (Saar 1988a). Indeed the optical results were uncertain enough (due to the low $f$ value) to be deemed a non-detection. The completely different, regression analysis approach of Mathys and Solanki (1988) yields $f$ and $B$ values suprisingly similar to Saar et al. (1986b), though the later recalibration (Mathys and Solanki 1989) produced magnetic field strengths larger than indicated by infrared data (Saar 1988a; Marcy and Basri 1989). The Marcy and Basri (1989) analysis includes improved radiative transfer and explicit disk-integration, and results in lower $B$ and larger $f$ values, though $f B$ is approximately the same. Thus, happily, there is now some convergence in the results for $\mathrm{fB}$ and $\sqrt{\mathrm{f}} \mathrm{B}$, but further work is needed to understand the why the separate $\mathrm{f}$ and $\mathrm{B}$ values differ. As noted in section 3, part of the disagreement in the derived $f$ and $B$ for $\epsilon$ Eri (and other targets) is probably due to the differing wavelengths and lines used, and part is due to the different analysis techniques themseves. Finally, one should remember that even in the best of cases, it is difficult to uniquely separate f and B (Gray 1984). 


\section{Some Future Directions}

What questions need to be addressed in stellar magnetic research over the next few years? First, many more magnetic measurements are needed. Determinations for more $M$ dwarfs and RS CVn's as well as first detections of T Tauri's and F dwarfs would be especially useful. Far infrared measurements of the 12 micron lines (Deming et al. 1988) and near infrared measurements using cryogenic echelles now being built will substantially increase the accuracy of the $f$ and $B$ determinations. More multiwavelength campaigns would help probe the temporal dependence of magnetic flux and its relationship to atmospheric heating. Observations of broadband linear (Huovelin et al. 1988) and circular (Kemp et al. 1987) polarization should help unravel some information on the spatial structure of the fields, given suitable theory (Landi Degl'Innocenti 1982; Mürset et al. 1988). It may be possible to obtain further spatial information through Stokes V (Donati et al. 1989) or Stokes I Doppler imaging (the spot profile "bumps" will be broader in high $\mathrm{g}_{\text {eff }}$ lines). Multiline observations and analyses (e.g., Mathys and Solanki 1989) should be further explored and refined. Observations over stellar cycle timescales should prove enlightening.

Several important assumptions have been made in stellar Zeeman analyses to date, and it is important to reassess them. The assumption of identical magnetic and non-magnetic atmospheres should eventually be discarded in favor of realistic flux-tube atmospheres for the magnetic component. Infrared data may require a third, umbral component (Sun et al. 1987). Turbulent and convective properties in magnetic regions appear to differ from the quiet solar atmosphere (Livingston 1982) and may in stars as well (Toner and Gray 1988). This should be investigated in detail. Magneto-optical effects should also be included in the modeling (Landolfi et al. 1989).

In summary, much has been learned in the past ten years about magnetic fields on solar-like stars, and their relationship to both basic stellar properties and to magneticallyrelated activity. Only the surface of the subject has been really explored, however, and many questions remain. Hopefully, ten years hence, many of these will also have been answered, bringing better understanding of the stellar "activity" phenomenon.

Acknowledgements. This work has been supported by the Smithsonian Institution postdoctoral research fellowship program, and NASA grants NAGW-221 and NGL-006-03057. I am very grateful to NOAO for the generous allocation of telescope time, and to the McMath stellar team in particular for all their help over the years. I would also like to thank G. Basri and G. Marcy for kindly sharing results with me prior to publication, and extend special thanks to many of the people referenced below for a multitude of stimulating discussions! 


\section{REFERENCES}

Ambruster, C. A., Saar S. H.: 1988, Bull. A. A. S., 20, 995

Basri, G. S. : 1987 Astrophys. J. 316, 377

Basri, G. S., Marcy, G. W. : 1988 Astrophys. J. 330, 274

Bohigas, J., Carrasco, L., Torres, C. A. O., Quast, G. R. : 1986 Astron. Astrophys. 157, 278

Bopp, B. W., Saar, S. H., Ambruster, C., Feldman, P., Dempsey, R., Allen, M., Barden, S. P. : 1989 Astrophys. J. 339, 1059

Borra, E. F., Edwards, G., Mayor, M. : 1984 Astrophys. J. 284, 211

Bruning, D. H. : 1984 Astrophys. J. 281, 830

Bruning, D. H., Chenoweth, R. E., Marcy G. W.: 1987, in Cool Stars, Stellar Systems, and the Sun eds. J. Linsky and R. E. Stencel, New York, p. 36

Bruning, D. H., Saar, S. H.: 1989, in Solar and Stellar Granulation, eds. R. J. Rutten and G. Severino, Kluwer, Dordrecht, p. 145

Deming, D., Boyle, R. J., Jennings, D. E., Wiedemann, G. : 1988 Astrophys. J. 333, 978

Donati, J. F., Semel, M., Praderie, F.: 1989, presented at IAU Colloq. 121, Inside the Sun.

Durney, B. R., Robinson, R. D. : 1982 Astrophys. J. 253, 290

Galloway, D. J., Weiss, N. O. : 1981 Astrophys. J. 243, 945

Giampapa, M. S. 1984, in Space Research Prospects in Stellar Activity and Variability, eds.

A. Mangeney and F. Praderie, Obs. de Paris, Meudon, p. 309

Giampapa, M. S. : 1985 Astrophys. J. 299, 781

Giampapa, M. S., Golub, L., Worden, S. P. Astrophys. J. Letters 83268121

Golub, L.: 1983, in Cool Stars, Stellar Systems, and the Sun eds. P. Byrne and M. Rodonó Reidel, Dordrecht, p. 83

Gondoin, Ph., Giampapa, M. S., Bookbinder, J. A. : 1985 Astrophys. J. 297, 710

Grossmann-Doerth, Larsson, B., ans Solanki, S. K. : 1988 Astron. Astrophys. 204, 266

Gray, D. F.: 1976, The Observation and Analysis of Stellar Photospheres, Wiley, New York

Gray, D. F. : 1984 Astrophys. J. 277, 640

Gray, D. F. : 1985 Publ. Astron. Soc. Pacific 97, 719

Gray, D. F.: 1988, Lectures on Spectral-line Analysis: F, G, and K Stars, The Publisher, Arva

Gray, D. F., Nagar, P. : 1985 Astrophys. J. 298, 756

Harvey, J. W., Hall, D. S.: Bull. A. A. S., 7, 459

Huovelin, J., Saar, S. H., Tuominen, I. : 1988 Astrophys. J. 329, 882

Jordan, C., Ayres, T. R. Brown, A., Linsky, J. L., Simon, T. : 1987 Mon. Not. R. Astr. Soc. 225, 903

Kawaler, S. D. : 1988 Astrophys. J. 333, 236

Kemp, J. C. et al. : 1987 Astrophys. J. (Letters) 317, L29

Kurucz, R. L. : 1979 Astrophys. J. Suppl. 40, 1

Landi Degl'Innocenti, E. : 1982 Astron. Astrophys. 110, 25

Landolfi, M., Landi Degl'Innocenti, M., Landi Degl'Innocenti, E. : 1989 Astron. Astro- 
phys. 216, 113

Linsky, J. L. : 1985 Solar Phys. 100, 333

Linsky, J. L.: 1989, Solar Phys., in press.

Linsky, J. L., Saar, S. H.: 1987, in Cool Stars, Stellar Systems, and the Sun eds. J. Linsky and R. E. Stencel, Springer, New York, p. 44

Livingston, W. C.: 1982, Nature, 297, 208

Marcy, G. W. : 1981 Astrophys. J. 245, 624

Marcy, G. W. : 1982 Publ. Astron. Soc. Pacific 94, 989

Marcy, G. W. : 1984 Astrophys. J. 276, 286

Marcy, G. W., Basri, G. S.: 1989, Astrophys. J. , 345, in press.

Marcy, G. W., Bruning, D. H. : 1984 Astrophys. J. 281, 286

Mathys, G., Solanki, S. K.: 1988, in The Impact of Very High S/N Spectroscopy on Stellar

Physics, ed. G. Cayrel de Strobel and M. Spite, Kluwer, Dordrecht, p. 325

Mathys, G., Solanki, S. K. : 1989 Astron. Astrophys. 208, 189

Montesinos, B., Fernandez-Figuerola, M. J., de Castro, E. K. : 1987 Mon. Not. R. Astr. Soc. 229, 627

Mould, J. R. : 1976 Astron. Astrophys. 48, 443

Mürset, U., Solanki, S. K., Stenflo, J. O. : 1988 Astron. Astrophys. 204, 279

Noyes, R. W., Hartmann, L. W., Baliunas, S. L., Duncan, D. K., Vaughan, A. H. : 1984 Astrophys. J. 279, 763

Pettersen, B. R. : 1989 Astron. Astrophys. 209, 279

Parker, E. N.: 1978, Ap. J., 221, 368

Quillen, A., Golub, L., Harnden, Jr., F. R., Saar, S. H.: 1987, Bull. A. A. S., 19, 1027

Robinson, R. D. : 1980 Astrophys. J. 239, 961

Robinson, R. D., Worden, S. P., Harvey, J. W. : 1980 Astrophys. J. (Letters) 236, L155

Saar, S. H.: 1987a, Observations and Analysis of Photospheric Magnetic Fields on G, K, and $M$ Dwarf Stars, Ph. D. thesis, University of Colorado

Saar, S. H.: 1987b, in Cool Stars, Stellar Systems, and the Sun eds. J. Linsky and R. E. Stencel, Springer, New York, p. 10

Saar, S. H. : 1988a Astrophys. J. 324, 441

Saar, S. H.: 1988b, in The Impact of Very High S/N Spectroscopy on Stellar Physics, ed.

G. Cayrel de Strobel and M. Spite, Kluwer, Dordrecht, p. 295

Saar, S. H.: 1988c, in Hot Thin Plasmas in Astrophysics, ed. R. Pallavicini, Kluwer, Dordrecht, p. 139

Saar, S. H.: 1989, in The Sun in Time, ed. M. Matthews, Tucson, U. of Arizona, in press

Saar, S.H., Huovelin, J., Giampapa, M.S., Linsky, J.L., Jordan, C.: 1988, in Activity in Cool Star Envelopes, Eds. O.Havnes et al., Kluwer, Dordrecht, p.45

Saar, S. H., Linsky, J. L. : 1985 Astrophys. J. (Letters) 299, L47

Saar, S. H., Linsky, J. L.: 1986a, Advances in Space Physics, 6, No. 8, 235

Saar, S. H., Linsky, J. L.: 1986b, in Cool Stars, Stellar Systems, and the Sun, eds. M. Zeilik and D. M. Gibson, Springer, New York, p. 278

Saar, S. H., Linsky, J. L., Beckers, J. M. : 1986a Astrophys. J. 302, 777

Saar, S. H., Linsky, J. L., Duncan, D. K.: 1986b, in Cool Stars, Stellar Systems, and the 
Sun, eds. M. Zeilik and D. M. Gibson, Springer, New York, p. 275

Saar, S. H., Linsky, J. L., Giampapa, M. S.: 1987, in 27th Liegé International Astrophysical Colloquium: Observational Astrophysics With High Precision Data, eds. L. Delbouille and A. Monfils, Universite de Liège, Liège, p. 103

Saar, S. H., Schrijver, C. J.: 1987, in Cool Stars, Stellar Systems, and the Sun, eds. J. Linsky and R. E. Stencel, Springer, New York, p. 38

Schatzman, E.: 1962, Ann. Astrophys., 25, 18

Schrijver, C., Coté, J., Zwaan, C., Saar, S.H.: 1989, Astrophys. J., 337, 964

Skumanich, A., MacGregor, K.: 1986, Advances in Space Physics, 6, No. 8, p. 151

Spruit, H. C., Zweibel, H. G. : 1979 Solar Phys. 628, 15

Stenflo, J. O. 1989, Astron. Astrophys. Rev., 1, 3

Steiner, O., Pneuman, G. W., Stenflo, J. O. : 1986 Astron. Astrophys. 170, 126

Stenflo, J. O., Lindegren, L. : 1977 Astron. Astrophys. 59, 367

Stepień, K. : 1987 Publ. Astron. Soc. Pacific 98, 1292

Stepień, K. : 1988a Astrophys. J. 335, 892

Stepień, K. : 1988b Astrophys. J. 335, 907

Stepień, K. : 1989 Astron. Astrophys. 210, 273

Stix, M.: : 1972 Astron. Astrophys. 20, 9

Sun, W.-H., Giampapa, M. S., Worden, S. P. : 1987 Astrophys. J. 312, 930

Tarbell, T. D., Title, A. M. : 1977 Solar Phys. 52, 13

Toner, C. G., Gray, D. F. : 1988 Astrophys. J. 334, 1008

Unno, W. 1956, Pub. Ast. Soc. Jap., 8, 108.

Vilhu, O. : 1984 Astron. Astrophys. 133, 117

Weber, E. J., Davis, L.: 1967, Ap. J., 148, 217

Zwaan, C., Cram, L. E.: 1989, chapter 7 in The Atmospheres of Cool Stars, eds. L. E. Cram and L. V. Kuhi, NASA-CNRS Monograph Series, in press 